\title{
A low-cost spatial contrast sensitivity display driver
}

\author{
WLLIAM B. CUSHMAN \\ Naval Aerospace Medical Research Laboratory, Pensacola, Florida
}

\begin{abstract}
The circuit described here can provide the line and frame signals to drive an $x, y$ display scope or oscilloscope at high resolution (1,000 lines) and repetition rate $(50 \mathrm{~Hz})$. Synchronizing circuitry at the input of the circuit allows the raster scan to be locked to an input signal, such as the squarewave output from a function generator, while another waveform from the same function generator, the sine output, drives the $z$ (intensity) input of the display scope. A stable spatial contrast display results. Spatial contrast depth is a direct function of $z$ input modulation voltage. Spatial frequency is a direct function of the function generator frequency. The circuit can be used with a programmable function generator under computer control.
\end{abstract}

Spatial contrast sensitivity is a widely used means of assessing basic visual performance. At the Naval Aerospace Medical Research Laboratory (NAMRL), the device described here has been used to assess visual degradation in humans while they are experiencing unusual acceleration and to assess visual degradation in rhesus monkeys while they are in strong electromagnetic fields. Available commercial devices are extremely expensive and often limited to the resolution of ordinary television monitors (roughly 250 lines/screen). If high spatial frequencies are to be tested with television-type monitors, the monitor must be placed at some distance from the subject to compensate for the lack of resolution. The circuit described here provides 1,000 lines per frame and can be viewed at close range without visible raster lines, thus allowing spatial contrast sensitivity experiments to be implemented in confined spaces. The frame repetition rate of the circuit is set at $50 \mathrm{~Hz}$ to prevent flickering of the image.

In experiments at NAMRL, we have used very high resolution $x, y$ display devices (Tektronix 606B) at close ranges (approximately $18 \mathrm{in}$.). The Tektronix 606B is far more precise than is required for spatial contrast sensitivity testing. Any reasonably good electrostatic $x, y$ display device would work as well if the input frequency response is at least $1 \mathrm{MHz}$. Sine- and square-wave (sync)

This research was supported by the Naval Medical Research and Development Command under work unit number 6152N MR00001.001. The author's contribution to this project was made during an Office of Naval Technology fellowship appointment at NAMRL. Trade names of materials and/or products of commercial or nongovernment organizations are cited as needed for precision. These citations do not constitute official endorsement or approval of the use of such commercial materials and/or products. Opinions and conclusions contained in this report are those of the author and do not necessarily reflect the views or the endorsement of the Department of the Navy, Department of Defense, or the U.S. Government. Address correspondence to the author at the Naval Aerospace Medical Research Laboratory, Naval Air Station, Pensacola, FL 32508-5700. signals were from a Keithley-Metrabyte PCIP-SST programmable function generator installed in an IBM-PCcompatible computer.

\section{Theory of Operation}

Except for phosphor persistence, only one spot of light is present at any given instant on a cathode-ray tube such as is used in an $x, y$ display monitor. If a spatial contrast sensitivity display is desired, this spot of light must be made to do several things. The screen must first be filled in. That is to say, the spot of light must pass across every location on the screen in a time frame short enough to be perceived as instantaneous, and then the whole process has to repeat at a frequency above the critical flicker fusion frequency of the subject. Passing the spot over the entire screen is usually accomplished with a raster scan. When using a raster scan, the spot starts at, for example, the top left part of the screen and proceeds from left to right at a fixed rate of speed. When the spot reaches the end of the line, it then returns at a very high rate back to the left side and starts over slightly below where it was before. In this manner, the entire screen is "painted." The circuit described here develops the line part of the raster scan by charging a capacitor through a constantcurrent diode. The voltage on the capacitor will increase linearly, and this voltage can then be used (after buffering) to drive the $x$ input of the display scope. A linear voltage will result in a linear displacement of the spot in the $x$ direction. The spot is then returned by shorting the capacitor to ground through two transistors in parallel and a diode.

The $y$ input voltage is developed in a similar fashion. A capacitor is linearly charged through a constant-current diode, and the voltage on the capacitor is buffered and fed directly to the $y$ input of the display scope, thus causing a deflection in the $y$ direction. The line and frame $(x$ and $y$ ) signals are synchronized by digitally counting the line signal down to zero, at which time three transistors in parallel with the frame capacitor are briefly shorted 


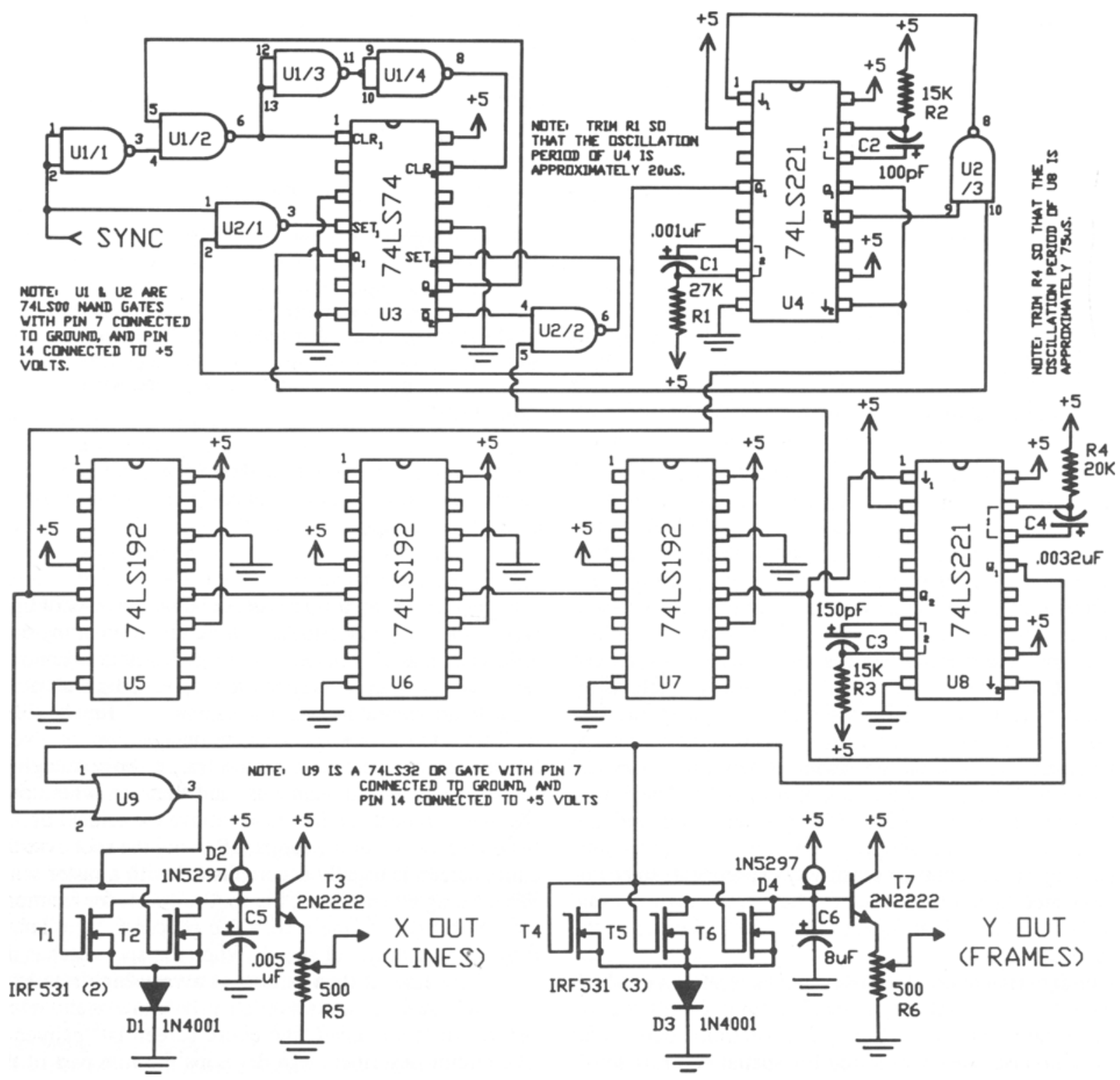

Figure 1. Schematic diagram of the contrast sensitivity display driver.

to ground, thus causing the spot to retrace. The whole process then repeats.

A sine-wave voltage from the function generator is connected to the $z$ input of the display scope to modulate the intensity of the spot. Timing is critical. The circuit has provision to detect the leading edge of a TTL-compatible signal (increasing from 0 to $2.5-5.0 \mathrm{~V}$ ) and to synchronize the raster scan to this event. Many function generators have separate sync outputs. If available, this sync output should be used. Even if the external sync function is not available, most function generators provide sine-, triangle-, and square-wave outputs that are internally synchronized. In this case, the square-wave output can be used as the synchronizing signal for the circuit described here, and the sine wave can be fed directly to the $z$ input of the display scope through an attenuator.

The circuit described here cannot work at all frequencies. There are small frequency windows during which the raster scan and sine frequency are in a harmonic relationship where jitter in the 74LS221 timing circuits causes synchronizing to become erratic. These frequency windows are extremely small and of no practical consequence with normal use.

\section{Detailed Circuit Description}

A schematic of the circuit is shown in Figure 1. If the leading edge of the sync signal goes positive and the not$\mathrm{Q}$ output of $\mathrm{U} 4$ pin 4 is high, then flip-flop number one 
of U3 is set by U2/1 causing the Q output of this flip-flop to be set high. A high signal from pin 5 of U3 causes U2/3 to go low if the not-Q output of U4 one-shot number two is in its resting state (high), thus starting one-shot number one of $U 4$. The $Q$ output of one-shot number one of U4 (pin 13) is connected to a decade counter, U5, and to pin 2 of OR gate U9. The output of U9, pin 3, causes the gates of $\mathrm{T} 1$ and $\mathrm{T} 2$ to be biased high and thus to short capacitor C5 to ground through D1. T3 is an emitter-follower buffer driving a potentiometer used to vary the output voltage of the line signal. When one-shot number one of $\mathrm{U} 4$ times out, the $\mathrm{Q}$ output (pin 13) drops to ground doing three things. First, the high at $\mathrm{U} 9$ pin 2 is removed, thus resulting in $\mathrm{T} 1$ and $\mathrm{T} 2$ being turned off, allowing C5 to linearly charge up through D2. Second, a count is completed at the input to decade counter U5. Third, oneshot number two of U4 is triggered (at pin 9), thus initiating the line interval. When one-shot number two times out, the not-Q output (pin 12) triggers one-shot number one via $\mathrm{U} 2 / 3$, and the process repeats until $\mathrm{U} 2 / 3$ pin 10 is brought low.

The output of decade counter U5 is fed directly to decade counter U6 and from there to decade counter U7, thus dividing the input pulse train by 1,000 . The output of U7 is fed directly to the inputs of one-shots one and two of U8. One-shot number one has a brief output pulse (about $75 \mu \mathrm{sec}$ ) from pin 13, which serves to bias transistors T4, T5, and T6 on, thus shorting capacitor C6 to ground through D3. At the same time, the output pulse from pin 13 U8 goes to pin 1 of U9 and causes T1 and $\mathrm{T} 2$ to be biased on, thus shorting $\mathrm{C} 5$ to ground through D1. By shorting both the line and frame capacitors simultaneously, the retrace path is forced along the bottom and left side of the display, where it is not visible. Transistor $\mathrm{T} 7$ is an emitter-follower similar to T3.

The output of one-shot number two of U8 at pin 5 is much briefer (about $1 \mu \mathrm{sec}$ ) than one-shot number one and is used to reset the synchronizing circuitry. The output from pin 5 of U8 goes high when one-shot number two is timing, causing pin 5 of $U 2 / 2$ to be high, and since flip-flop number two of $\mathrm{U} 3$ is cleared (as will become evident below), the not-Q output is also high, causing $U 2 / 2$ to go low and set flip-flop number two, thus removing the high from pin 8. When flip-flop number two is set, the $Q$ output goes high (pin 9). Assuming at least one pe- riod of the sync positive-going signal has passed, making the output of $U 1 / 1$ high, U1/2 will then clear flipflop number one. After two gate delays provided by $U 1 / 3$ and U1/4, flip-flop number two will clear. At this point, the whole process can repeat.

\section{Concluding Remarks}

The circuit described here should be constructed in a metal box with ventilation to allow cooling of the components and power supply. Use good layout techniques, and bypass all integrated circuit power pins (pin 14 or 16) with $0.1 \mu \mathrm{F}$ ceramic capacitors to ground. The power supply must provide at least $200 \mathrm{~mA}$ at $5 \mathrm{~V}$. BNC connectors and heavy, short coaxial cables should be used to connect the circuit to the display monitor. Thin or long coaxial cables have high capacitance and will not pass high frequencies. If strange, ghostlike images or lines occur, the most likely problem is ringing in the coaxial cables. Keep them under $1 \mathrm{~m}$ in length, if possible.

Using an oscilloscope, adjust R5 and R6 to produce peak-to-peak voltages of $1 \mathrm{~V}$ at the outputs, or whatever voltage your display scope inputs require. The ramps seen on the oscilloscope should be linear and have very fast return times. The offset controls on the $z$ input of the display scope should be adjusted so that the output light level with no modulation is the same as the average of the peak and trough light levels with modulation. To be precise, make this adjustment with a photometer.

The $x$ and $y$ inputs to the display scope can be reversed to produce a vertically oriented spatial contrast sensitivity display or left as they are for a horizontal display. A two-interval forced-choice experimental paradigm based on display orientation could be easily implemented with the addition of suitable switching. Or, a two-interval paradigm can be implemented by presenting the display versus no display.

The cost of parts varies widely depending on the source used, but should be under $\$ 25$ for the devices shown in Figure 1. Additional parts such as a box, cord, connectors, switch, and power supply will bring the total cost to roughly $\$ 75$.

(Manuscript received March 25, 1991; revision accepted for publication January 9, 1992.) 